\title{
LocationWeb: Proposal and Implementation of Location-based Web Content Search and Creation Using a Mobile Phone
}

\author{
Misato Sasaki, ${ }^{\dagger}$ Christian Noack,${ }^{\dagger \dagger}$ Hidetoshi Yokota $^{\dagger}$ \\ and AKIRA IDOUE ${ }^{\dagger}$
}

\begin{abstract}
As location positioning systems such as GPS become popular, there is a growing demand for location-based applications. It is getting easier to utilize map information by connecting a GPS receiver to a PC and PDA. Corresponding to this momentum, GPS receivers embedded into mobile phones and applications using the user location in real-time are widely available. Location information is also gaining attention as a form of meta-data for web content and is applied to the semantic web, which provides advanced search services using meta-data information. Taking advantages of these situations, the creation of web content "on the spot" in a flexible form on a mobile phone is becoming important. In this paper, we propose a system called "LocationWeb", which provides location-based web content search and creation on the mobile phone. This uses the location information as meta-data of the web content and enables handy search services based on real time location information.
\end{abstract}

\section{Introduction}

Recently, applications combined with location information such as vehicle and personal navigation systems have attracted attention. A personal navigation agent proposed by Refs. 1), 2) provides information spontaneously according to the location of the user, where PHS is used for wireless communication and positioning of the user location. A similar personal agent proposed by Ref.3) obtains location information maintained by a mobile network operator via JAIN and PARLAY APIs and provides appropriate services for the user. Location information adds value to other content information such as advertisements of restaurants or shops. Furthermore, adding location information to a picture ${ }^{4)}$ or to an e-mail is also becoming common. Such services are currently used within small groups of people such as friends or family. However, there are also attempts to open public location information for unspecified groups of people. Mobile phones equipped with digital cameras have become widespread, facilitating the creation of content with such photos and uploading them on the spot, such as moblogs ${ }^{5}$ ).

If it is possible to attach location information to the content, it can be used as a key for search and as meta-data for the semantic

$\dagger$ KDDI R\&D Laboratories, Inc.

$\dagger \dagger$ Brandenburg University of Technology Cottbus, Germany web ${ }^{6)}$. This latter is an extension of the current web, in which information is given welldefined meaning, further assisting collaboration between computers and people ${ }^{7}$. Information associated with the content is represented as meta-data and by attaching such information to the web content, more precise and useful services can be provided. For example, GeoURL is a database system for registering latitude and longitude and showing specific positions on a map ${ }^{8)}$. Also, RDFIG ${ }^{9)}$ attempts to represent geographical location in RDF (Resource Description Format) ${ }^{10)}$. However, the location information must be obtained using a special device, or the position must be obtained by other means such as using a web site that provides the latitude and longitude by specifying the point in the map. There are some web sites that offer search based on location information. They present search results close to the position by providing location information such as an address and keywords for the search ${ }^{11)}$. The targets of these sites are, however, usually for information on fixed objects such as restaurants and hotels. Another type of service provides the location of the nearest land mark, expresses the location information in the form of a URL and then shows it on the map. If the search target is a moving object, however, their location information must be updated to provide timely information. In this situation, positioning and content searching devices both need to be portable. With the reduction in the cost of GPS receivers, connecting one to a notebook 
$\mathrm{PC}$ or PDA becomes easier, thus spawning further research work using these positioning devices. For example, equipping a PDA with a GPS receiver and indicating the individual location information of a group is useful when meeting up. Some groupware using location information has been studied ${ }^{12}$ ).

Equipping PDAs with GPS receivers and installing driver software, however, is timeconsuming and troublesome for the general public who are unfamiliar with computing devices. On the other hand, a growing number of GPS equipped mobile phones enable a variety of useful location related applications at their fingertips. There are already several map applications using a mobile phone's GPS function. However, the appearance and GUI interface for inputting information are prepared in advance and the liberty of formatting is limited; even in moblogs. People usually visit web sites from a desktop PC at their homes or offices. However, when the user is in a mobile environment, it is difficult to reflect the location information or to renew the content. By enabling the real time location to be uploaded and creation of content "on the spot", we can expect a greater variety of location-based services. To realize these features, we propose LocationWeb, which is an enhanced location-based service platform in a mobile environment.

\section{Proposal of an Integrated Web Ap- plication Based on Location Infor- mation}

The discussion in the previous section motivates us to adopt the design principle that the following functions are realized "on the spot":

(1) Creating and editing web content,

(2) Uploading location information,

(3) Searching function using user location information, and

(4) Displaying the search results on the screen.

We propose a system that coordinates an application to edit content in real-time on a mobile phone and one to search web sites by interpreting the semantic of the location. Figure 1 describes the concept of the LocationWeb. LocationWeb is based on three basic factors, namely the Internet, mobile phones and GPS. Combining these factors, we construct a handy system that can create and search web

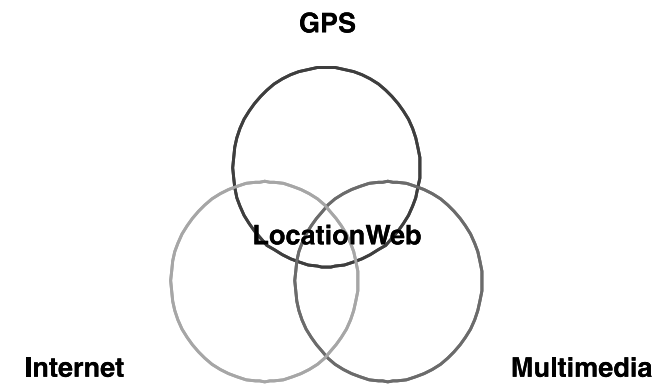

Fig. 1 The concept of LocationWeb.

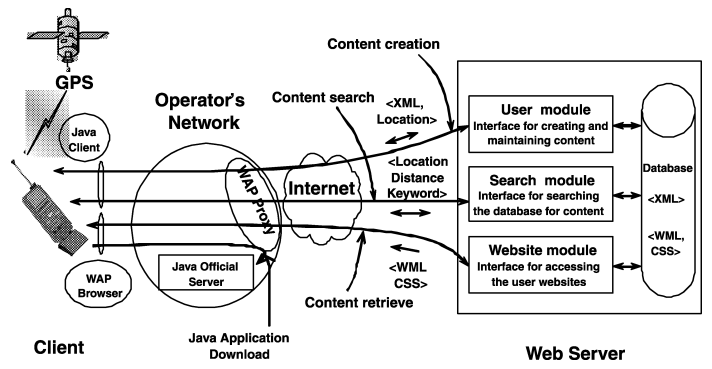

Fig. 2 The overall structure of the proposed system.

content using location information.

In this paper, we also propose a mobile phone application based on LocationWeb, which provides location semantic combination web search services. Mobile phones are here equipped with the function of capturing location information and creating and searching web content using captured location information.

\subsection{Overview and Structure of the Proposed system}

The structure of the proposed system is shown in Fig. 2. The mobile phone on the user side is first connected to the operator's network and accesses the web server via the Internet. The operator's network provides several functions such as a WAP proxy to translate HTML to WML for WAP browsers or the java official server to download approved and clean java applications.

The proposed web server consists of the following web applications:

(1) A user module to create and store contents,

(2) A search module to search the content database,

(3) A web site module to respond to requests from the client for web contents.

On the other hand, the user accesses the web server with a mobile phone equipped with a function to acquire location information, a java virtual machine and a WAP browser. Data ex- 


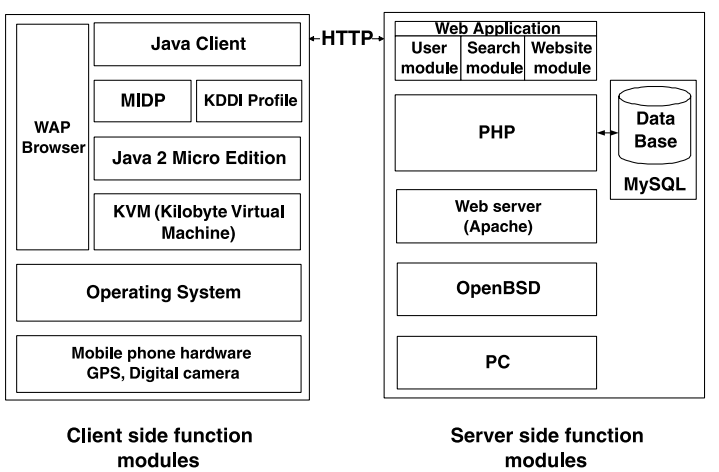

Fig. 3 Structure of the functional modules.

change between the user module on the server side and the Java client on the user side is conducted into an XML ${ }^{13)}$ format. The user module translates the XML format to the WML (Wireless Markup Language) ${ }^{14)} /$ CSS (Cascading Style Sheet) ${ }^{15)}$ format for the mobile phone to browse, and stores both formats in the database. The search module provides a function to use the user location and a geographical range as a search condition in addition to keywords in text format. The web site module obtains the requested web content from the database, and transfers it to the mobile phone in the WML format.

The structure of the functional modules in the proposed system is shown in Fig. 3. We used apache (Version 2.0.4.8) for the web server, MySQL (Version 4.0.16) for the DBMS and PHP (Version 4.3.4) for coordinating both entities. In addition, we used J2ME for the java application runtime environment on the mobile phone, MIDP, which is a mobile phone profile and KDDI-P, which is also a profile provided by KDDI for GPS functions. For communications between the Java client or the WAP browser on the client side and the web applications on the web server side, HTTP is both supported and used.

\subsection{Structure of the Client Side}

The package structure of the functional modules on the client side is shown in Fig. 4. The main module handles the registration procedure for opening a web site and the builder module accesses the user module on the server side to manage the web site and cards, which are the content units, as well as setting up the location information. The drawer module provides a web editor function, which is the interface used to make content using the mobile phone operating buttons. Created web contents are then

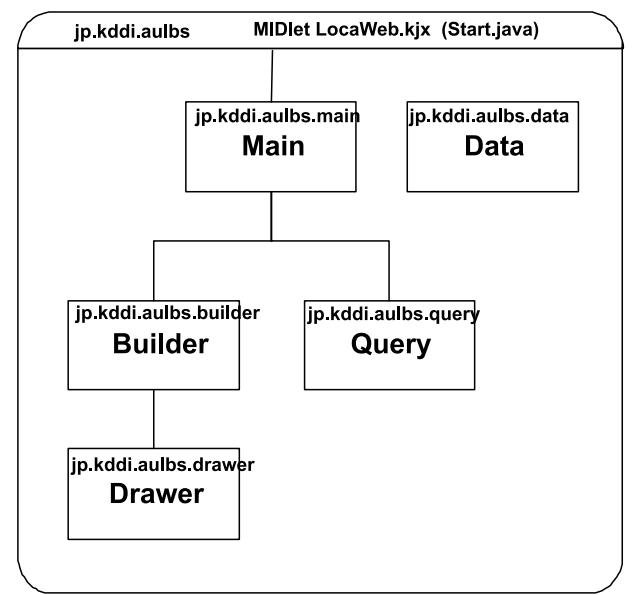

Fig. 4 The package construction for function modules.

stored in the XML format. The query module provides a form for content search and enables the following function. Connecting with the search module, the search result is plotted in the form of coordinates on the display. The data module controls data access inside the mobile phone. This makes it possible to access data such as pictures stored in the data folder and also to capture location information using GPS. To avoid accessing the server every time the user makes and renews content, new content is kept in the internal mobile phone cache. When making content, the information in this cache is first surveyed, and if it has not already been stored there, the server is then accessed.

There are two ways of realizing the above operation environment on the client side. One is to prepare an application for web content editing on a mobile phone. The other is a method like CGI, where the client receives an HTML form from a web server and uploads it every time. The client application size in this system is about $95 \mathrm{Kbytes}$, and the average time taken to download the application from the java official server was $9.3 \mathrm{sec}$ when using an actual mobile phone (Kyocera W11K). The size of an application that could be performed on a recent mobile phone is sufficiently large and the time for downloading an application, which is needed only for the first time, was within $10 \mathrm{sec}$. We considered adopting the former method using a java application.

On the other hand, the latter method can be used also on a PC with a web browser and is therefore considered to be effective when a PC is used in combination with a mobile phone. 


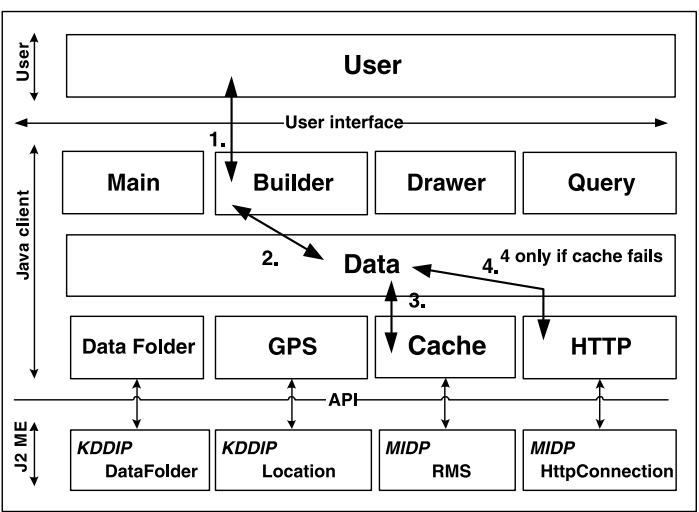

Fig. 5 Layered representation of the function modules (obtaining data).

However, a device for obtaining the location information needs to be prepared. The function modules shown in Fig. 4 are illustrated in a layered fashion in Fig. 5. Accessing the data folder inside the mobile phone, obtaining the location or cache information and the HTTP transfer function are performed by the Java client program via the APIs provided by J2ME. These types of information are solely managed by the data module, which bridges them to the main, builder, drawer and query modules. When the user requests data to create content, the request is received by the builder module with button operations (1), and handed to the data module (2). The data module first searches the RMS (Record Management System) provided by J2ME to see if it is stored in the cache (3). If no corresponding information is found, the data module obtains the necessary information from the web server via HTTP transfer (4).

As shown in Fig. 6, when uploading the created web content, the user request will be transferred to the data module via the builder module (1), and only the modified part, including all the materials used to construct the content, is transferred from the data folder (2). At the same time, the information transferred to the web server is also cached in the RMS (3). From this time on, when content data are needed (4), those inside the cache are used (5).

\subsection{Structure of the Server Side}

Figure 7 shows a series of procedures for translating and storing content on the server side. XML content sent from the client, is distinguished by the subscriber ID, which is attached to the HTTP request header. It is then translated into WML and stored in the

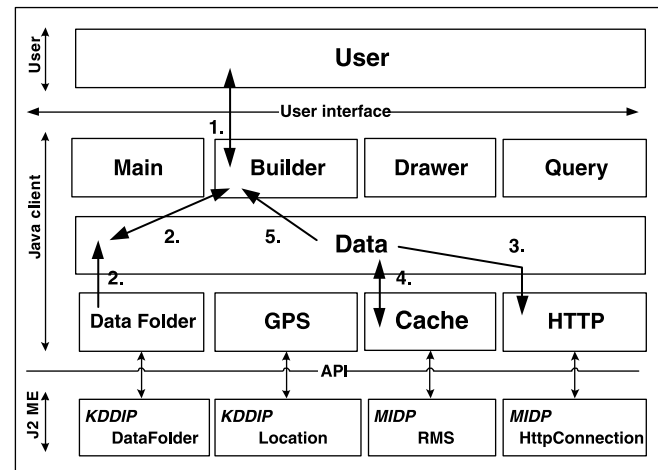

Fig. 6 Layered representation of the function modules (renewing data).

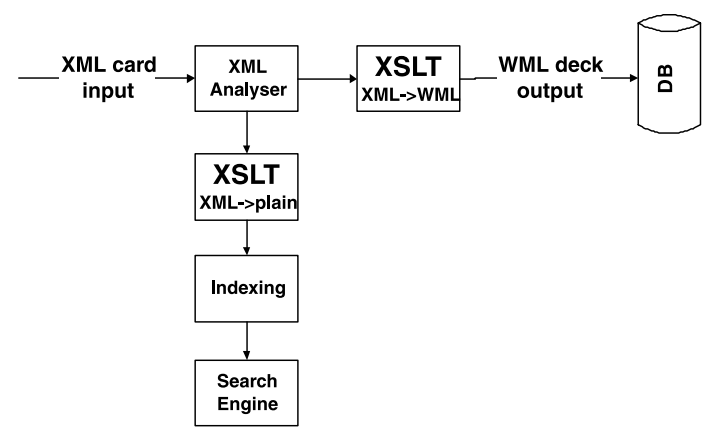

Fig. 7 Content input and storage procedures on the server side.

database to support WAP browsers. Content information from the user is also stored in the search database. In the proposed system, tags are stripped off the text, from which each word is then extracted and stored in the search table by linking it with the ID of the WML card that includes the word (Indexing). To perform the above transfer, we adopted the XSLT (eXtensible Stylesheet Language Translator) ${ }^{16)}$ program. For the search method, we use two functions: keyword and search range measured from the user location. By showing results that meet these two functions, the web content can be selected.

\section{Overview of the Proposed System Operations}

In this section, we present the operation and user interface of the proposed system.

\subsection{Creation of Web Content}

The user first downloads the Java application from a specific server and launches it. Materials for web content are created by inputting text information or pictures taken by the mobile phone and saved in the data folder. By selecting 


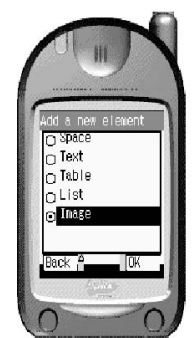

(a)

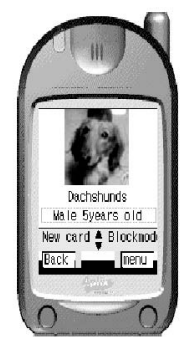

(b)

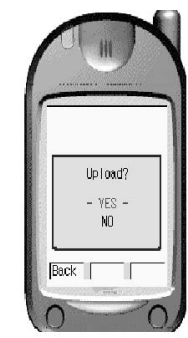

(c)
Fig. 8 Web content creation interface.

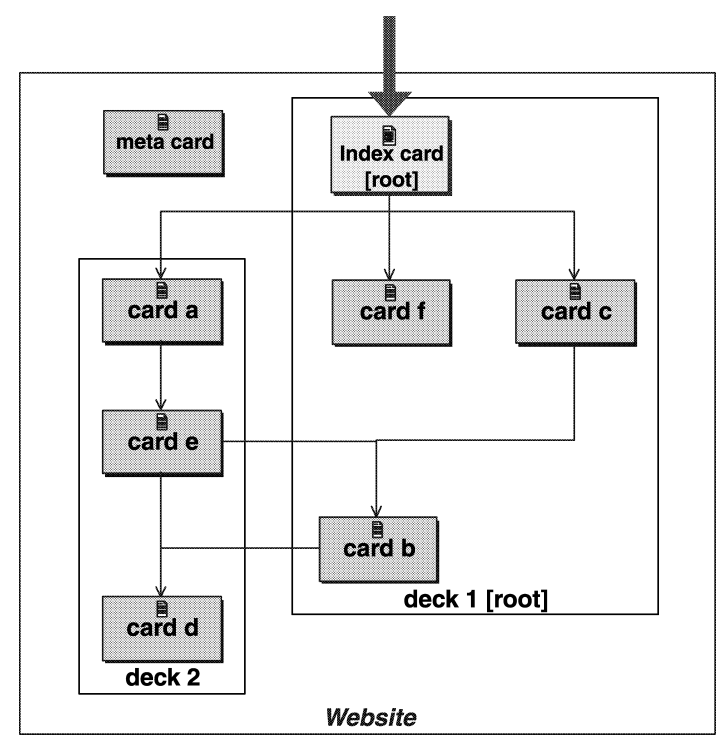

Fig. 9 Creation of web content and site.

the picture data (Fig. 8 a), the web content can be displayed and edited on the screen (Fig. 8 b). Subsequently, the user accesses the proposed web server and uploads the created web content in XML format with the user's location information (Fig. 8c). On the web server side, it is translated into WML format and stored together with the XML format. With regard to the editing operations on the screen, text, line breaks, creation of tables including alignment and list making can all be performed in a WYSIWYG manner, which makes created content comparable to ones created on PC.

Each user is assigned a web site, where the created content is stored. The web site has a title, description, short name and location. The web content stored in the web site consists of one or more deck(s) as shown in Fig. 9. A deck is a collection of cards and a card is a unit of user interaction (e.g., a choice menu, a screen of text or a text entry field) written in XML

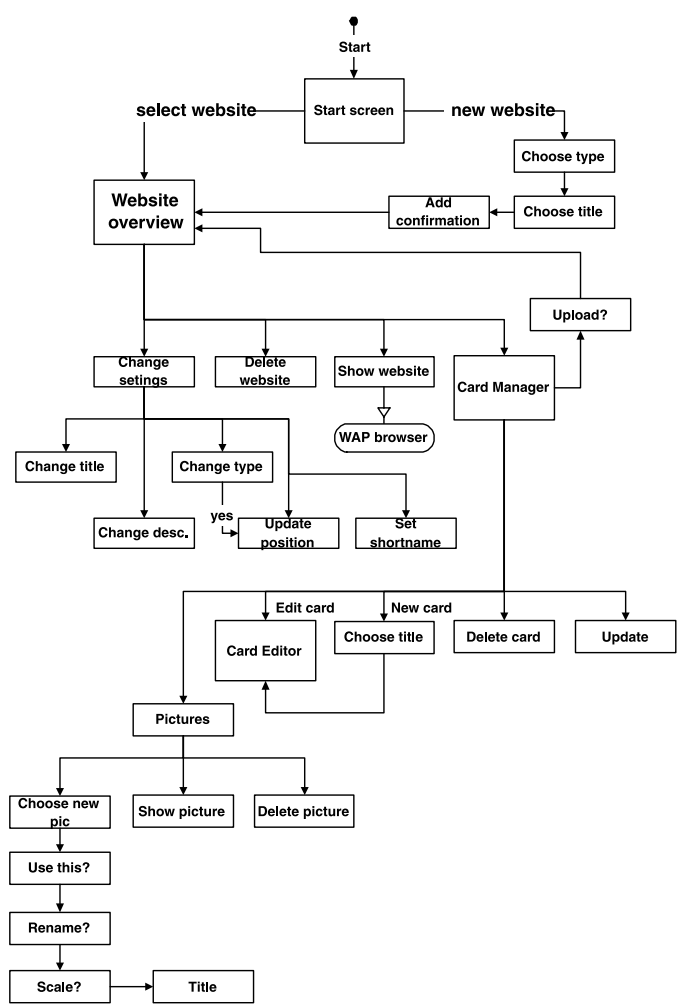

Fig. 10 Web content creation flowchart.

format.

Figure 10 shows a process flowchart for creating new web content. By following this process, the user can create, update and view the web site and web content. This system also enables web content renewal and the uploading of new location information.

\subsection{Search of Web Content And Re- sult}

To search web content, the user initially accesses the web server and chooses the search operation (Fig. 11 a). They then input keywords and the search range of the information desired (Fig. 11 b). The location information of the user is also attached to the above request and forwarded to the web server; the result is shown around the user's real-time location (Fig. 11 c). By selecting from the search results shown, the desired information concerning the web content can be obtained by the button operations. The web content information will then appear on the screen.

In this way, since creating, searching and browsing web content can all be performed using a mobile phone, providing a handy and portable application services becomes easy. 
Table 1 Operation time measurement.

\begin{tabular}{c|c|c|c|c}
\hline \hline Operation & $\begin{array}{c}\text { Overall operation time } \\
(\mathrm{ms})\end{array}$ & $\begin{array}{c}\text { Communication time } \\
(\mathrm{ms})\end{array}$ & $\begin{array}{c}\text { Request data size } \\
\text { (byte) }\end{array}$ & $\begin{array}{c}\text { Response data size } \\
\text { (byte) }\end{array}$ \\
\hline Upload web content & 2,413 & 309 & 1,503 & 222 \\
Update location & 2,230 & 272 & 1,332 & 166 \\
Search for content & 2,112 & 287 & 1,361 & 644 \\
Browse search result & 3,093 & 349 & 2,432 & 618 \\
\hline
\end{tabular}

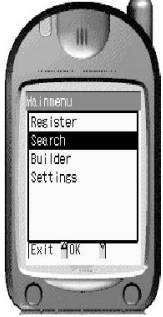

(a)

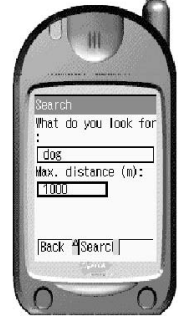

(b)

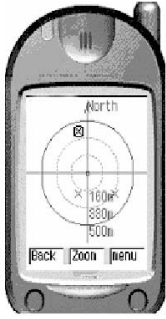

(c)
Fig. 11 Searching and inspecting the screen.

One of the features in the proposed system is the ability to upload location information on demand. The proposed system, however, does not allow the transmission of location information when the user does not intend to do so. If the user is located where the GPS signal is inaccessible or opts to save battery, the location information is not updated; although the contents are stored in the web server and accessible at any time. The usage of the location information connected to the content depends on how the user uses the system and the timeliness of the location information is totally controlled by the user.

To show the handiness of the proposed application, we measure the time for the four major operations: uploading the created content, updating the location, searching for the content and browsing the search result. The request and response data sizes depend on the content and we used one sample for uploading and browsing, and three samples for searching. The measurement results are shown in Table 1. The overall operation time is measured on the mobile phone, which is what the user experiences, and the communication time is defined as the time between the start and end of the TCP connection(s) for one operation. We measure each operation 10 times and the listed values are the average of them. With regard to the time for updating the location and searching for the content, the time for positioning is not included. From the results, each operation takes about 2 to 3 seconds and we can say it is roughly comparable to the case when $\mathrm{PC}$ is

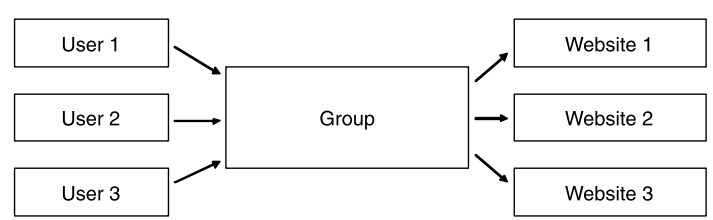

Fig. 12 Image of group forming in LocationWeb.

used for the same operations.

\section{Discussion}

In order to identify each mobile user, a subscriber ID is used. This is a unique ID given to each mobile phone. By using this subscriber ID, it is possible to limit access to the web content. This is attached to the HTTP request header. The format of the subscriber ID is composed of the following elements:

XXXXnnnnnnnnnn_[host name].[domain name]

(i) (ii) (iii) (iv)

\section{(i) Area ID code (4 7 digits)}

(ii) Mobile code ( $7 \sim 10$ digits $)$, unique ID given to the server

(iii) "_": Underscore

(iv) Host name (e.g., ma)

(v) Domain name (e.g., ezweb.ne.jp)

The application presented in this paper showed how location is utilized to find the information that is physically close to an individual user in real-time. If the location information is further shared with other people, locationbased groupware can also be realized. In such case, the web site is supposed to be accessed by multiple users forming a group and therefore one-to-one binding of a subscriber ID and a web site needs to be extended to allow for a group-based access as shown in Fig. 12.

According to the increase in the amount of web content, the importance of the searching method will also increase. A more efficient and context matched search will be provided by a combination of location and text information. Further research is required, however, to accelerate the search analysis and make it more accurate when the amount of content increases. The prototype system stores content information in both XML and WML formats to take the con- 
nection with the mobile phone into consideration. By further supporting RDF, the proposed system will become a more effective semantic web system by jointly working with other web systems.

\section{Conclusions}

We developed a system of creating and searching through web content based on location information using mobile phones, and suggested new methods of communicating based on location. The increase in the amount of information available makes it difficult to find that which is really needed, and the demand for essential and desired information from extraneous information is likely to increase. The proposed system will provide an effective method to create and search information on a real-time location basis and will extend the potential of a mobile phone and its applications.

\section{References}

1) Kase, N., et al.: Yugashiman(1)-Why Agentcan make a Human Navigation System?, 61st IPSJ National Convention, pp.3-379-3380 (Oct. 2000).

2) Kase, N., et al.: Yugashiman(2)-An Implementation of a Human Navigation System, 61st IPSJ Nationnal Convention, pp.3-381-3382 (Oct. 2000).

3) Cheng, S.-T., et al.: A New Framework for Mobile Web Services, pp.218-222, SAINT'02 (Jan. 2002).

4) Ricoh GPS compatible RDC-i700G: http://www.dpreview.com/news/0302/ 03022601ricohi700g.asp

5) Moblog: http://en.wikipedia.org/wiki/Moblog

6) W3C - Semantic Web: http://www.w3.org/2001/sw/

7) Berners-Lee, et al.: The Semantic Web, Scientific American, Vol.284, No.5, pp.34-43 (May 2001).

8) GeoURL: http://geourl.org/

9) Brickley, D.: RDFIG Geo Vocab Workspace, http://www.w3.org/2003/01/geo/

10) W3C - RDF: http://www.w3.org/RDF/

11) Google Local: http://local.google.com/lochp

12) Yoshino, T., et al.: Development of LocationAware Collaboration System with Communication Functions of One Another's Situation, Vol.2002, No.24, pp.20-25, IPSJ SIG Tech. Report (Mar. 2002).

13) W3C - XML: http://www.w3.org/XML/

14) WAP Forum: Wireless Application Protocol
Wireless Markup Language Specification, version 1.1 (June 1999).

15) W3C - CSS:

http://www.w3.org/Style/CSS/

16) W3C - XSLT:

http://www.w3.org/Style/XSL/

(Received May 31, 2005)

(Accepted September 2, 2005)

(Released November 9, 2005)

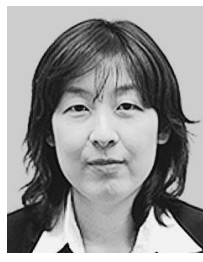

Misato Sasaki received the B.E. degree from Seikei University and M.E. degree from Tokyo Institute of Technology University, Japan, in 2001 and 2003, respectively. She joined KDDI R\&D Laboratories, Inc., Japan, in 2003. Her current research interests include mobile communication.

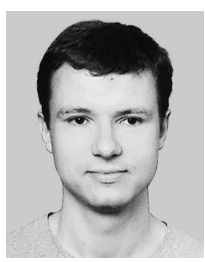

Christian Noack is a student from Germany, studying Computer Science at the Brandenburg University of Technology, Cottbus. From 2003 to 2004, he attended an 6 month internship in the Mobile Network Group of KDDI R\&D Laboratories, Inc., Japan. His interests are network applications and security.

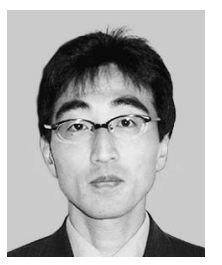

Hidetoshi Yokota received the B.E., M.E. and Ph.D. degrees from Waseda University, Tokyo, in 1990, 1992 and 2003, respectively. He joined KDDI R\&D Laboratories, Inc., Japan, in 1992. From 1995 to 1996 he was with SRI International, Menlo Park, CA as an International Fellow. He received the Young Engineer Award in 1998 from IEICE. His current research interests include mobile communications and Internet QoS. He is a member of IPSJ, IEICE and IEEE. 


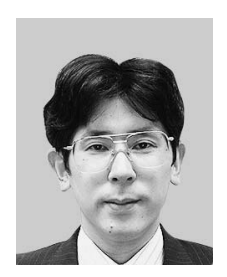

Akira Idoue received the B.E. and M.E. degrees of electrical engineering from Kobe University in 1984 and 1986 respectively. Since joining KDD in 1986, he worked in the field of computer communication. His current research interests include high performance communication protocols, protocol testing and mobile computing. He is currently a senior manager of Mobile Network Lab. in KDDI R\&D Laboratories, Inc. He received IPSJ Convention Award in 1992 and Best Paper Award of IPSJ National Convention in 1998. 